Published in final edited form as:

Arch Ophthalmol. 2010 April ; 128(4): 443-447. doi:10.1001/archophthalmol.2010.31.

\title{
Longitudinal Postnatal Weight Measurements for the Prediction of Retinopathy of Prematurity
}

\author{
Dr. Carolyn Wu, MD, \\ Departments of Ophthalmology, Children's Hospital Boston, Harvard Medical School, Boston, \\ Massachusetts \\ Dr. Deborah K. VanderVeen, MD, \\ Departments of Ophthalmology, Children's Hospital Boston, Harvard Medical School, Boston, \\ Massachusetts
}

Dr. Ann Hellström, MD, PhD, Institute of Neuroscience and Physiology, Sahlgrenska Academy at University of Gothenburg, Göteborg, Sweden

Dr. Chatarina Löfqvist, $\mathrm{PhD}$, and Institute of Neuroscience and Physiology, Sahlgrenska Academy at University of Gothenburg, Göteborg, Sweden

\author{
Dr. Lois E. H. Smith, MD, PhD \\ Departments of Ophthalmology, Children's Hospital Boston, Harvard Medical School, Boston, \\ Massachusetts
}

\section{Abstract}

Objective-To validate longitudinal postnatal weight gain as a method for predicting severe retinopathy of prematurity (ROP) in a US cohort.

Methods-Both ROP evaluations and weekly weight measurements from birth to postmenstrual week 36 for 318 infants were entered into a computer-based surveillance system, WINROP. This system signaled an alarm when the rate of weight gain decreased compared with control subjects. Infants were classified into 3 groups: (1) no alarm, (2) low-risk alarm, or (3) high-risk alarm. Maximum ROP for each infant was categorized as (1) no ROP (immature or mature vascularization), (2) mild ROP (stage 1 or 2 ROP in zone II or III, without plus disease), or (3) severe ROP (any prethreshold, any stage 3, or threshold ROP). A high-risk alarm identified infants at risk for developing severe ROP.

Results-A high-risk alarm occurred in 81 infants (25.5\%) and detected all infants who developed severe ROP a median of 9 weeks before diagnosis. The remaining infants received no alarm or a low-risk alarm. None of these infants developed more than mild ROP.

(C)2010 American Medical Association. All rights reserved.

Correspondence: Carolyn Wu, MD, Department of Ophthalmology, Children's Hospital Boston, 300 Longwood Ave, Boston, MA 02115 (carolyn.wu@ childrens.harvard.edu).

Financial Disclosure: PremaCure AB has rights to the WINROP system; Drs Hellström and Löfqvist own shares in a company controlling PremaCure AB. 
Conclusions-Longitudinal postnatal weight gain may help predict ROP. In a US cohort, the WINROP system had a sensitivity of $100 \%$ and identified infants early who developed severe ROP. With further validation, WINROP has the potential to safely reduce the number of ROP examinations.

Retinopathy of prematurity (ROP) is a neovascular eye disease that affects the developing retina in premature infants and is a leading cause of preventable childhood blindness in developed and developing countries. ${ }^{1-3}$ Most cases of ROP are mild and spontaneously regress without treatment. However, severe cases of ROP lead to progressive retinal scarring and detachment, resulting in vision loss. Detection and treatment of ROP through screening have been shown to decrease the incidence of severe vision loss and to reduce unfavorable outcomes. ${ }^{4,5}$

Screening for ROP is performed in infants with a birth weight (BW) of less than $1500 \mathrm{~g}$ or a gestational age (GA) of 30 weeks or younger and in select infants with a BW of 1500 to $2000 \mathrm{~g}$ with an unstable clinical course who are believed to be at high risk for ROP. ${ }^{6}$ Screening for ROP often requires multiple examinations using indirect ophthalmoscopy with scleral depression. These examinations are stressful for infants, ${ }^{7-9}$ labor intensive for the ophthalmologist, and often unavailable in remote or underserved areas. Based on the revised guidelines from the Early Treatment for Retinopathy of Prematurity study, ${ }^{5}$ approximately $8 \%$ of screened infants will require treatment for ROP. Since 1981, the rate of premature birth in the United States has risen more than $36 \%$, from $9.4 \%$ to $12.8 \%,{ }^{10}$ leading to more infants qualifying for screening to identify less than $10 \%$ of infants who will require treatment. ${ }^{5}$ Given the low treatment yield of all screened infants, more cost-effective programs are needed to identify infants at high risk for developing severe ROP. Current ROP screening guidelines do not consider post-natal clinical risk factors that may affect the development of ROP. ${ }^{1,11,12}$

The weight, insulinlike growth factor (IGF), neonatal ROP (WINROP) algorithm is an online surveillance system developed in Sweden to evaluate the risk of severe ROP based on weekly postnatal measurements of serum IGF-1 levels and weight. ${ }^{13}$ This system is designed to detect a slowing of the rise in an infant's IGF-1 levels or a slowing of weight gain compared with expected values in a cohort of premature infants who developed no ROP or only stage 1 ROP. ${ }^{13}$

Several studies ${ }^{13-15}$ have validated this algorithm. In the initial study, ${ }^{13}$ the WINROP algorithm was developed and tested using data from 79 preterm infants at risk for ROP (ie, an in-sample evaluation). Using the WINROP system, 23\% of infants were considered at risk for developing treatable ROP. All 6 infants who required treatment were correctly identified at least 5 weeks before treatment. A second prospective study ${ }^{15}$ was performed on a new population of 50 infants from another region in Sweden. The system identified all 9 infants who developed proliferative ROP an average of 9 weeks (range, 1.1-21.6 weeks) before diagnosis.

These studies used IGF-1 levels and weight gain to predict ROP. Excluding IGF-1 levels from this algorithm would simplify the screening to weight measurements alone(which are 
easily and routinely obtained), would reduce the cost and stress on infants from blood sample collections, and may potentially be available in underserved areas with limited resources. A retrospective study ${ }^{14}$ of 353 children in Sweden was performed using the WINROP algorithm based on postnatal weight gain alone. Eighty-five of these children (24.1\%) were identified before 32 weeks' postmenstrual age to be at risk for developing treatable ROP. All of the infants who developed proliferative ROP $(n=35,41.2 \%)$ and all who required treatment $(n=25,29.4 \%)$ were correctly identified in this group. The median time from the alarm to treatment was 9 weeks (range, 4.0-18.3 weeks). The objective of the present study was to evaluate whether this algorithm also provided early prediction of the development of severe ROP in a population of premature infants in the United States.

\section{METHODS \\ PATIENTS}

A retrospective review was performed on all premature infants born at the Brigham and Women's Hospital between October 21, 2005, and December 12, 2008, who qualified for ROP screening. Infants who were born at a GA older than 32 weeks or who did not have weekly weight measurements available until 36 weeks' postmenstrual age because of discharge or transfer to another facility, other than Children's Hospital Boston, were excluded from this study. Infants were required to have ROP follow-up until ROP was regressing, mature retinal vascularization or immature retinal vascularization in zone III was reached, prethreshold or threshold ROP occurred, or treatment was required. The following data were collected: GA at birth, BW, weekly postnatal weight measurements until 36 postmenstrual weeks, sex, parity, race, ROP examination results, and the incidences of bronchopulmonary dysplasia, intraventricular hemorrhage, hydrocephalus, and necrotizing enterocolitis.

\section{ROP SCREENING AND TREATMENT}

All the infants were examined according to standard ROP protocol consisting of a dilated funduscopic examination using binocular indirect ophthalmoscopy with scleral depression. The first ROP examination was performed between 30 and 34 postmenstrual weeks based on GA at birth: 6 weeks of age for GA of 26 weeks or younger, 5 weeks of age for GA of 27 to 28 weeks, 4 weeks of age for GA of 29 to 30 weeks, and 3 weeks of age for GA of 31 to 32 weeks. Examinations usually occurred every 1 to 2 weeks but ranged from twice per week to every 3 weeks depending on the severity and zone of ROP. The highest stage of ROP and lowest zone were recorded, as was the presence of prethreshold or threshold disease and the need for treatment.

Retinopathy of prematurity was classified according to the International Classification of Retinopathy of Prematurity (stages 1-5). ${ }^{16}$ Threshold ROP is defined as 5 contiguous or 8 cumulative clock hours of stage 3 ROP with plus disease in zone I or II. Prethreshold disease is defined as any zone I ROP, zone II stage 2 ROP with plus disease, or zone II stage 3 ROP without plus disease or with plus disease but less than the requisite clock hours to qualify as threshold disease. Based on the Early Treatment for Retinopathy of Prematurity study criteria, ${ }^{5}$ standard treatment with laser photocoagulation was provided if ROP became 
severe enough to warrant treatment at type 1 ROP. Type 1 ROP is defined as any zone I ROP with plus disease, zone I stage 3 ROP without plus disease, or zone II stage 2 or 3 ROP with plus disease.

For ROP analysis in this study, the maximum ROP for the worse eye was calculated for each infant and was categorized into 3 groups: (1) no ROP (immature or mature vascularization), (2) mild ROP (stage 1 or 2 ROP in zone II or III, without plus disease), or (3) severe ROP (any prethreshold, any stage 3, or threshold ROP). Infants who required treatment for ROP were further analyzed separately as a subset of patients with severe ROP.

\section{WINROP SCREENING}

The WINROP algorithm was developed using the methods of online statistical surveillance. ${ }^{17-19}$ A reference model of the expected weekly IGF-1 levels and weight gain was developed using data from infants who developed no ROP or stage 1 ROP. ${ }^{13}$ The differences between the weekly expected postnatal IGF-1 level and weight gain and the observed values are calculated and accumulated. When the accumulated sum exceeds a limit, an alarm is signaled to indicate that the infant may be at risk for developing severe ROP. In this study, only postnatal weight gain was evaluated using the WINROP algorithm.

Each infant's GA, BW, and weekly postnatal weight from birth to postmenstrual week 36 were entered into the algorithm. When an alarm was signaled, the infant's GA at birth and $\mathrm{BW}$ were reevaluated to determine whether the infant was at low or high risk for developing severe ROP. High-risk infants were classified as having a GA at birth of younger than 30 weeks and a BW of less than $850 \mathrm{~g}$. Infants were classified into 3 alarm levels: (1) no alarm, (2) low-risk alarm, or (3) high-risk alarm.

\section{STATISTICAL ANALYSIS}

Each infant's alarm risk was compared with his or her maximum ROP group. We evaluated the ability of the WINROP algorithm to detect all cases of severe ROP with a high-risk alarm signal. The sensitivity and specificity of the WINROP screening using the variables of GA at birth, BW, and weekly post-natal weight gain were analyzed. The negative and positive predictive values were calculated using the sensitivity, specificity, and prevalence of severe ROP for the study group. We calculated $95 \%$ confidence intervals for estimated binary proportions (sensitivity and specificity) by using the exact method by ClopperPearson. ${ }^{20}$

\section{RESULTS}

PATIENTS

For this study, 361 infants were eligible. Forty-three infants were excluded (Table 1). Thirty-three excluded infants were born at a GA older than 32 weeks, and none developed ROP. Nine infants were excluded because of missing weights. Weights were missing owing to unavailability of the medical record for 2 infants, and weights were not recorded at the specified weekly interval for 7 infants who were transferred to Children's Hospital Boston for surgical care. Two of these 9 infants developed prethreshold ROP, 1 of whom was 
treated, and the other 7 infants developed no more than mild ROP. One additional infant who developed prethreshold ROP was excluded from the study because of nonphysiologic excessive weight gain after complications from gastrointestinal surgery.

A total of 318 infants participated in the study. The median GA was 29 weeks (range, 23-32 weeks), and the median BW was $1050 \mathrm{~g}$ (range, 450-2400 g). Male infants accounted for $55 \%$ of the study population, and 181 infants were singleton births, 118 were twin births, and 19 were triplet births. The study population was $60 \%$ white, $19 \%$ black, $13 \%$ Hispanic, and $8 \%$ Asian. There was a higher rate of complicating factors, such as bronchopulmonary dysplasia, intraventricular hemorrhage, hydrocephalus, and necrotizing enterocolitis, in infants with more severe ROP (Table 2).

\section{ROP AND WINROP OUTCOMES}

Severe ROP developed in 28 infants (8.8\%), with 13 (4.1\%) requiring treatment. No ROP developed in 171 infants (53.8\%), and 119 infants (37.4\%) developed mild ROP (Table 3).

No alarm was signaled in 56 infants, and a low-risk alarm was signaled in 181 infants. None of the infants in these groups developed more than mild ROP, and all regressed without treatment (Table 3). The median GA at birth was 29 weeks (range, 25-31 weeks) in the no alarm group and 30 weeks (range, 25-32 weeks) in the low-risk alarm group. The median BW was $1450 \mathrm{~g}$ (range, 820-2400 g) in the no alarm group and $1118 \mathrm{~g}$ (range, 840-2090 g) in the low-risk alarm group.

A high-risk alarm was signaled in 81 infants (25.5\%). All 28 infants who developed severe ROP were identified in this alarm group. The median GA at birth was 25 weeks (range, 2330 weeks), and the median BW was $770 \mathrm{~g}$ (range, 450-850 g) in this group. Thirteen infants in this group required treatment for ROP. The median GA at birth was 24 weeks (range, 2327 weeks), and the median BW was $640 \mathrm{~g}$ (range, 490-830 g) in infants who required treatment for ROP.

For the 28 infants who developed severe ROP, the median time from birth to the alarm signal was 3 weeks (range, 1-11 weeks) and from alarm to the development of severe ROP was 9 weeks (range, $1-14$ weeks). The median time from the alarm signal to treatment was 8 weeks (range, 4-12 weeks) in the 13 infants who required treatment.

Using the WINROP algorithm with postnatal weight measurements in this US cohort, 81 infants $(25.5 \%)$ would require ophthalmologic examinations, thereby safely reducing the number of ROP examinations in $75 \%$ of the infants in this population. The forewarning given by the alarm did not reduce the number of examinations for the infants at risk, but it gave ample time for warning that ophthalmologic examinations should take place.

\section{TEST CHARACTERISTICS}

The sensitivity of the WINROP algorithm in detecting severe ROP was 100\% (95\% confidence interval, $87.7 \%-100 \%$; 28 of 28 infants) and the specificity was $81.7 \%$ (95\% confidence interval, $76.8 \%-86.0 \% ; 237$ of 290 infants). The positive predictive value was $34.6 \%$ (28 of 81 infants), and the negative predictive value was 100\% (237 of 237 infants). 


\section{COMMENT}

Current ROP screening guidelines use GA at birth and BW as criteria for screening. However, more than $90 \%$ of these infants will not require treatment, with ROP either regressing or never developing. ${ }^{5,21}$ With the increasing survival rate in premature infants and the decreasing number of ophthalmologists screening for ROP, more infants qualify for screening and are subject to multiple eye examinations, sometimes as frequently as every few days, creating an excess workload on ophthalmologists for identifying the few infants who will develop treatable ROP.

Retinopathy of prematurity is caused by abnormal post-natal retinal development. Identifying postnatal factors that may have a role in the incidence and severity of ROP would be beneficial for the screening and prevention of ROP. Low serum IGF-1 levels and poor postnatal weight gain are associated with the development of worse ROP in clinical and animal studies. ${ }^{13-15,22-26}$ The WINROP algorithm can use both of these factors to identify infants who are at high risk for developing severe ROP, or it can use postnatal weight gain alone. In retrospective and prospective studies ${ }^{13,15}$ in Sweden, the WINROP surveillance system accurately identified all infants who developed proliferative ROP. Further simplification of the WINROP system using only postnatal weight measurements has been shown to successfully identify all infants who will develop severe ROP requiring treatment and to correctly predict most infants who did not require treatment in a population of premature infants from Sweden. ${ }^{14}$ The present findings in the United States also support this simplified screening method, and we found similar results with $100 \%$ sensitivity and a $100 \%$ negative predictive value in detecting severe ROP. Whereas the Swedish population may represent a relatively homogeneous group for race, the present study population was more racially diverse.

In infants who developed severe ROP, the high-risk alarm was signaled a median of 3 weeks after birth, 9 weeks before the diagnosis of severe ROP, and 8 weeks before treatment for ROP. Identification of infants at risk for developing severe ROP weeks to months before onset may have a significant positive effect on the care of these infants. Because the development of treatable ROP can range from as early as 31 postmenstrual weeks to as late as several weeks after term, knowledge of ROP risk would optimize inpatient and outpatient management. Although we did not find that earlier ophthalmologic examinations were needed to detect treatable ROP, some of the more fragile premature infants require deferment or rescheduling of eye examinations because of medical instability. Information related to the ROP risk in these infants would be useful in the timing of follow-up ROP examinations. Some infants will develop treatable ROP after discharge from the neonatal intensive care unit, and outpatient follow-up is important. More regulated follow-up measures can be used for these high-risk infants to ensure visit compliance and decrease the risk of an unfavorable outcome from missed ROP appointments. The need for continued ROP screening may necessitate that some infants remain in the neonatal intensive care unit rather than being discharged or transferred to other neonatal intensive care units where treatment for ROP may not be readily available. In addition to targeting infants at higher risk, there would be a decrease in the number of stressful eye examinations performed on premature infants with no alarm and low-risk alarms. 
In this study, infants who developed severe ROP had a higher incidence of bronchopulmonary dysplasia, intraventricular hemorrhage, hydrocephalus, and necrotizing enterocolitis compared with those who developed no ROP or mild ROP. This is not surprising because this outcome follows with clinical observation that sicker and smaller infants develop more severe disease. Given the high rate of comorbidities associated with prematurity, care must be taken to exclude infants from the WINROP algorithm who develop nonphysiologic weight gain, such as from severe hydrocephalus or excessive edema.

Timely detection and treatment of ROP to obtain the best visual and structural outcome in premature infants who develop severe ROP are the foundation of ROP screening. New approaches using postnatal risk factors may help reduce the number of infants requiring eye examinations for ROP and may target infants at high risk for developing severe ROP. To be effective, these methods must be cost-efficient, be readily available, and have extraordinarily high sensitivity to detect treatable ROP. The WINROP algorithm using postnatal weight measurements seems to fulfill these conditions and has been validated in different patient populations in 2 countries. Continued validation with patient populations across the world is necessary before adopting new screening guidelines for ROP.

\section{References}

1. Gilbert C, Fielder A, Gordillo L, et al. International NO-ROP Group. Characteristics of infants with severe retinopathy of prematurity in countries with low, moderate, and high levels of development: implications for screening programs. Pediatrics. 2005; 115(5):e518-e525. http:// pediatrics.aappublications.org/cgi/content/full/115/5/e518. [PubMed: 15805336]

2. Maida JM, Mathers K, Alley CL. Pediatric ophthalmology in the developing world. Curr Opin Ophthalmol. 2008; 19(5):403-408. [PubMed: 18772673]

3. Muñoz B, West SK. Blindness and visual impairment in the Americas and the Caribbean. Br J Ophthalmol. 2002; 86(5):498-504. [PubMed: 11973241]

4. Cryotherapy for Retinopathy of Prematurity Cooperative Group. Multicenter Trial of Cryotherapy for Retinopathy of Prematurity: ophthalmological outcomes at 10 years. Arch Ophthalmol. 2001; 119(8):1110-1118. [PubMed: 11483076]

5. Early Treatment for Retinopathy of Prematurity Cooperative Group. Revised indications for the treatment of retinopathy of prematurity: results of the early treatment for retinopathy of prematurity randomized trial. Arch Ophthalmol. 2003; 121(12):1684-1694. [PubMed: 14662586]

6. Section on Ophthalmology American Academy of Pediatrics; American Academy of Ophthalmology; American Association for Pediatric Ophthalmology and Strabismus; American Academy of Ophthalmology; American Association for Pediatric Ophthalmology and Strabismus. Screening examination of premature infants for retinopathy of prematurity [published correction appears in Pediatrics. 2006;118(3):1324]. Pediatrics. 2006; 117(2):572-576. [PubMed: 16452383]

7. Clarke WN, Hodges E, Noel LP, Roberts D, Coneys M. The oculocardiac reflex during ophthalmoscopy in premature infants. Am J Ophthalmol. 1985; 99(6):649-651. [PubMed: 4014388]

8. Kleberg A, Warren I, Norman E, et al. Lower stress responses after Newborn Individualized Developmental Care and Assessment Program care during eye screening examinations for retinopathy of prematurity: a randomized study. Pediatrics. 2008; 121(5):e1267-e1278. [PubMed: 18450869]

9. Laws DE, Morton C, Weindling M, Clark D. Systemic effects of screening for retinopathy of prematurity. Br J Ophthalmol. 1996; 80(5):425-428. [PubMed: 8695564]

10. Martin JA, Hamilton BE, Sutton PD, et al. Births: final data for 2006. Natl Vital Stat Rep. 2009; 57(7):1-104. 
11. Fielder AR, Gilbert C, Quinn G. Can ROP blindness be eliminated? Biol Neonate. 2005; 88(2):98100. [PubMed: 15855745]

12. Gilbert C. Retinopathy of prematurity: a global perspective of the epidemics, population of babies at risk and implications for control. Early Hum Dev. 2008; 84 (2):77-82. [PubMed: 18234457]

13. Löfqvist C, Andersson E, Sigurdsson J, et al. Longitudinal postnatal weight and insulin-like growth factor I measurements in the prediction of retinopathy of prematurity. Arch Ophthalmol. 2006; 124(12):1711-1718. [PubMed: 17159030]

14. Hellström A, Hård AL, Engström E, et al. Early weight gain predicts retinopathy in preterm infants: new, simple, efficient approach to screening. Pediatrics. 2009; 123(4):e638-e645. [PubMed: 19289449]

15. Löfqvist C, Hansen-Pupp I, Andersson E, et al. Validation of a new retinopathy of prematurity screening method monitoring longitudinal postnatal weight and insulinlike growth factor I. Arch Ophthalmol. 2009; 127(5):622-627. [PubMed: 19433710]

16. Committee for the Classification of Retinopathy of Prematurity. An international classification of retinopathy of prematurity. Arch Ophthalmol. 1984; 102(8):1130-1134. [PubMed: 6547831]

17. Frisén M. Statistical surveillance: optimality and methods. Int Stat Rev. 2003; 71 (2):403-404.

18. Roberts S. A comparison of some control chart procedures. Technometrics. 1966; 8(3):411-430.

19. Shiryaev A. On optimum methods in quickest detection problems. Theory Probab Appl. 1963; 8:22-46.

20. Hollander, M.; Wolfe, D. Nonparametric Statistial Methods. 2. New York, NY: John Wiley \& Sons; 1999.

21. Palmer EA, Flynn JT, Hardy RJ, et al. Cryotherapy for Retinopathy of Prematurity Cooperative Group. Incidence and early course of retinopathy of prematurity. Ophthalmology. 1991; 98(11): 1628-1640. [PubMed: 1800923]

22. Allegaert K, Vanhole C, Casteels I, et al. Perinatal growth characteristics and associated risk of developing threshold retinopathy of prematurity. J AAPOS. 2003; 7(1):34-37. [PubMed: 12690367]

23. Engström E, Niklasson A, Wikland KA, Ewald U, Hellström A. The role of maternal factors, postnatal nutrition, weight gain, and gender in regulation of serum IGF-I among preterm infants. Pediatr Res. 2005; 57(4):605-610. [PubMed: 15695599]

24. Hellström A, Engström E, Hård AL, et al. Postnatal serum insulin-like growth factor I deficiency is associated with retinopathy of prematurity and other complications of premature birth. Pediatrics. 2003; 112(5):1016-1020. [PubMed: 14595040]

25. Hellström A, Perruzzi C, Ju M, et al. Low IGF-I suppresses VEGF-survival signaling in retinal endothelial cells: direct correlation with clinical retinopathy of prematurity. Proc Natl Acad Sci U S A. 2001; 98(10):5804-5808. [PubMed: 11331770]

26. Wallace DK, Kylstra JA, Phillips SJ, Hall JG. Poor postnatal weight gain: a risk factor for severe retinopathy of prematurity. J AAPOS. 2000; 4(6):343-347. [PubMed: 11124668] 


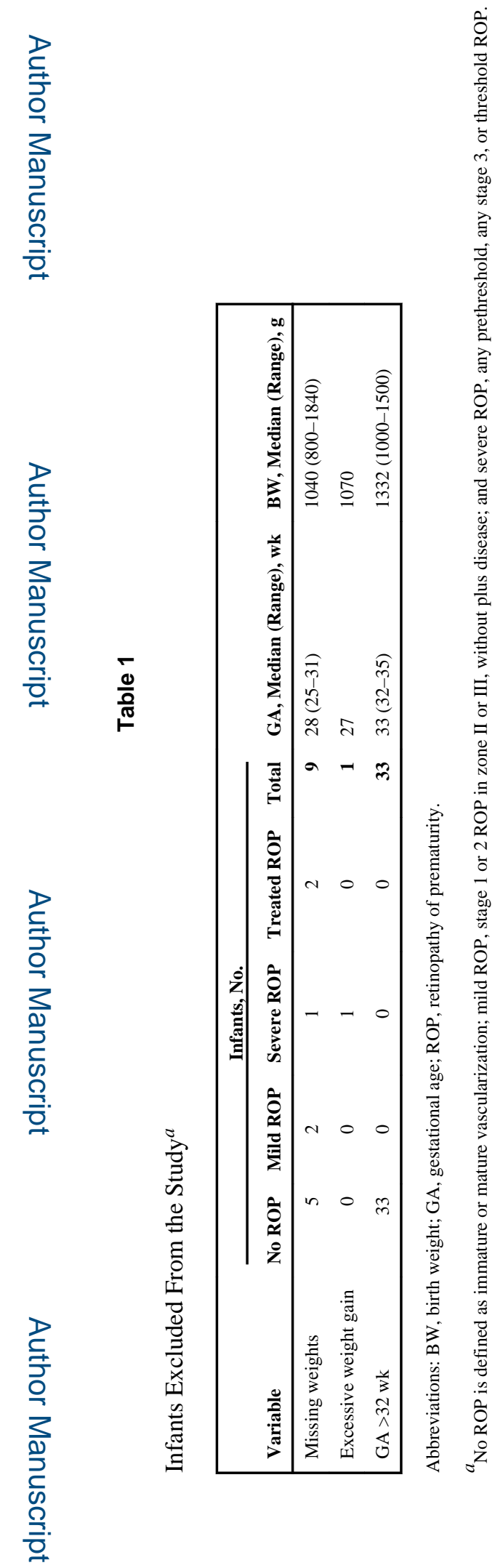

Arch Ophthalmol. Author manuscript; available in PMC 2015 April 11. 


\section{Table 2}

Prematurity-Associated Illnesses ${ }^{a}$

\begin{tabular}{|lcccc|}
\hline & \multicolumn{4}{c|}{ Infants, No. (\%) } \\
\cline { 2 - 5 } & Total (n=318) & No or Mild ROP $(\mathbf{n = 2 9 0})$ & Severe ROP (n=28) & Treated ROP (n=13) \\
\hline Bronchopulmonary dysplasia & $\mathbf{1 6 0}(50.3)$ & $133(45.9)$ & $27(96.4)$ & $13(100)$ \\
Intraventricular hemorrhage & $\mathbf{7 8}(24.5)$ & $62(21.4)$ & $16(57.1)$ & $10(76.9)$ \\
Hydrocephalus & $\mathbf{6}(1.9)$ & $5(1.7)$ & $1(3.6)$ & 0 \\
Necrotizing enterocolitis & $\mathbf{3 5}(11.0)$ & $28(9.7)$ & $7(25.0)$ & $5(38.5)$ \\
\hline
\end{tabular}

Abbreviation: ROP, retinopathy of prematurity.

${ }^{a}$ No ROP is defined as immature or mature vascularization; mild ROP, stage 1 or 2 ROP in zone II or III, without plus disease; and severe ROP, any prethreshold, any stage 3, or threshold ROP. 

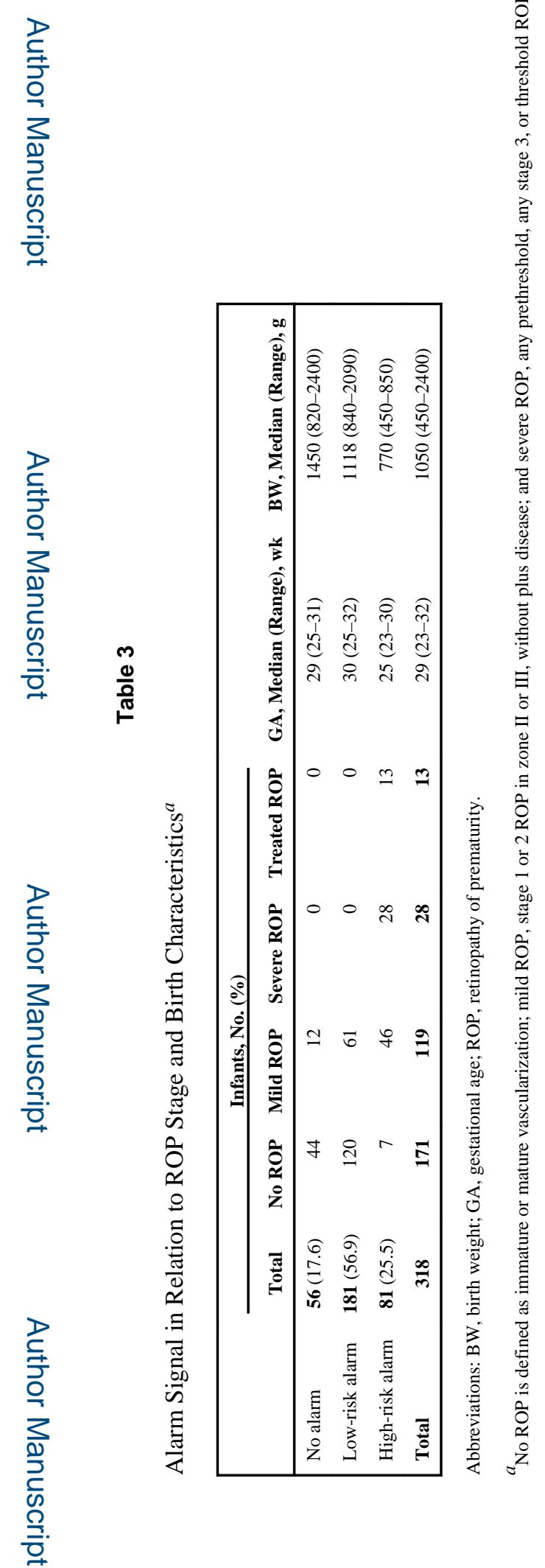

Arch Ophthalmol. Author manuscript; available in PMC 2015 April 11. 